Supporting Information

\title{
Short-Term Effects of Particle Sizes and Constituents on Blood Biomarkers among Healthy Young Adults in Guangzhou, China
}

Dan Feng ${ }^{1, \S}, \mathrm{Ke} \mathrm{Cao}^{1, \S}$, Zhi-Zhou He ${ }^{1, \S}$, Luke D. Knibbs², Bin Jalaludin ${ }^{3,4}$, Ari Leskinen ${ }^{5,6}$, Marjut Roponen $^{7}$, Mika Komppula ${ }^{5}$, Pasi Jalava ${ }^{7}$, Peng-Yue Guo ${ }^{1}$, Shu-Li Xu ${ }^{1}$, Bo-Yi Yang ${ }^{1}$, Liwen $\mathrm{Hu}^{1}$, Xiao-Wen Zeng ${ }^{1}$, Gongbo Chen ${ }^{1}$, Hong-Yao Yu ${ }^{1}$, Lizi Lin ${ }^{1}$, Guanghui Dong ${ }^{1, *}$

${ }^{1}$ Guangdong Provincial Engineering Technology Research Center of Environmental Pollution and Health Risk Assessment, Department of Occupational and Environmental Health, School of Public Health, Sun Yat-sen University, Guangzhou 510080, China

${ }^{2}$ School of Public Health, The University of Queensland, Herston, Queensland, 4006, Australia

${ }^{3}$ Centre for Air Quality and Health Research and Evaluation, Glebe, NSW, 2037, Australia ${ }^{4}$ IIngham Institute for Applied Medial Research, University of New South Wales, Sydney, 2170, Australia

${ }^{5}$ Finnish Meteorological Institute, Kuopio, 70211, Finland

${ }^{6}$ Department of Applied Physics, University of Eastern Finland, Kuopio, 70211, Finland

${ }^{7}$ Department of Environmental and Biological Sciences, University of Eastern Finland, Kuopio, FI, 70211, Finland

\section{*Address correspondence to:}

Guanghui Dong, MD, PhD, Professor, Guangdong Provincial Engineering Technology Research Center of Environmental Pollution and Health Risk Assessment, Department of Occupational and Environmental Health, School of Public Health, Sun Yat-sen University, 74 Zhongshan $2^{\text {nd }}$ Road, Yuexiu District, Guangzhou 510080, China. Phone: +862087333409 ; Fax: +862087330446. Email:donggh5@mail.sysu.edu.cn; donggh512@,hotmail.com

§These authors contributed equally to this work.

PAGES: 12

SUPPLEMENTAL TABLES: 8

SUPPLEMENTAL FIGURES: 1 


\section{Table of Contents}

Table S1. Spearman correlations between different blood biomarkers. (Page S3)

Table S2. Spearman correlations between size-fractionated PM and weather conditions. (Page S4)

Table S3. Spearman correlations between $\mathrm{PM}_{2.5}$ constituents. (Page S5-S6)

Table S4. Percentage changes (mean and 95\% confidence intervals) in CRP associated with interquartile range increases in 24-h average concentrations of $\mathrm{PM}_{2.5}$ constituents. (Page S7)

Table S5. Percentage changes (mean and 95\% confidence intervals) in MCP-1 associated with interquartile range increases in 24-h average concentrations of $\mathrm{PM}_{2.5}$ constituents. (Page S8)

Table S6. Percentage changes (mean and 95\% confidence intervals) in sVCAM-1 associated with interquartile range increases in 24-h average concentrations of $\mathrm{PM}_{2.5}$ constituents. (Page S9)

Table S7. Percentage changes (mean and 95\% confidence intervals) in sICAM-1 associated with interquartile range increases in 24-h average concentrations of $\mathrm{PM}_{2.5}$ constituents. (Page S10)

Table S8. Percentage changes (mean and 95\% confidence intervals) in ET-1 associated with interquartile range increases in 24-h average concentrations of $\mathrm{PM}_{2.5}$ constituents. (Page $\mathrm{S} 11$ ) Figure S1. Time series of size-fractionated PM and weather conditions in Guangzhou, China, between December 1, 2017 and January 5, 2018. (Page S12) 
Table S1. Spearman correlations between different blood biomarkers.

\begin{tabular}{lccccc}
\hline & CRP & MCP-1 & sVCAM-1 & sICAM-1 & ET-1 \\
\hline CRP & 1 & & & & \\
MCP-1 & $\mathbf{0 . 0 9}$ & 1 & & & \\
sVCAM-1 & 0.03 & $\mathbf{0 . 1 3}$ & 1 & & \\
sICAM-1 & $\mathbf{0 . 2 0}$ & 0.01 & $\mathbf{0 . 1 1}$ & 1 & \\
ET-1 & 0.05 & $\mathbf{0 . 2 0}$ & 0.09 & 0.04 & 1 \\
\hline
\end{tabular}

Abbreviations: CRP, C-reactive protein; ET-1, endothelin-1; sICAM-1, soluble intercellular adhesion molecule-1; MCP-1, monocyte chemoattractant protein-1; sVCAM-1, soluble vascular cell adhesion molecule-1.

Bold indicates $p<0.05$. 
Table S2. Spearman correlations between size-fractionated PM and weather conditions.

\begin{tabular}{|c|c|c|c|c|c|c|c|}
\hline & $\mathrm{PM}_{2.5}$ & $\mathrm{PM}_{1}$ & $\mathrm{PM}_{0.5}$ & $\mathrm{PN}_{0.2}$ & $\mathrm{PN}_{0.1}$ & Temperature & Relative humidity \\
\hline $\mathrm{PM}_{2.5}$ & 1 & & & & & & \\
\hline $\mathrm{PM}_{1}$ & 1.00 & 1 & & & & & \\
\hline $\mathrm{PM}_{0.5}$ & 0.99 & 0.99 & 1 & & & & \\
\hline $\mathrm{PN}_{0.2}$ & 0.31 & $\mathbf{0 . 3 0}$ & 0.34 & 1 & & & \\
\hline $\mathrm{PN}_{0.1}$ & 0.18 & 0.17 & 0.20 & 0.98 & 1 & & \\
\hline Temperature & 0.27 & 0.29 & 0.33 & 0.42 & 0.40 & 1 & \\
\hline Relative humidity & 0.12 & 0.14 & 0.16 & -0.12 & -0.14 & 0.17 & 1 \\
\hline
\end{tabular}

Abbreviations: $\mathrm{PM}_{2.5}$, particles with diameters $\leq 2.5 \mu \mathrm{m} ; \mathrm{PM}_{1}$, particles with diameters $\leq 1.0 \mu \mathrm{m} ; \mathrm{PM}_{0.5}$, particles with diameters $\leq 0.5 \mu \mathrm{m} ; \mathrm{PN}_{0.2}$, particles with diameters $\leq 0.2 \mu \mathrm{m} ; \mathrm{PN}_{0.1}$, particles with diameters $\leq 0.1 \mu \mathrm{m}$.

Bold indicates $p<0.0001$. 
Table S3. Spearman correlations between $\mathrm{PM}_{2.5}$ constituents.

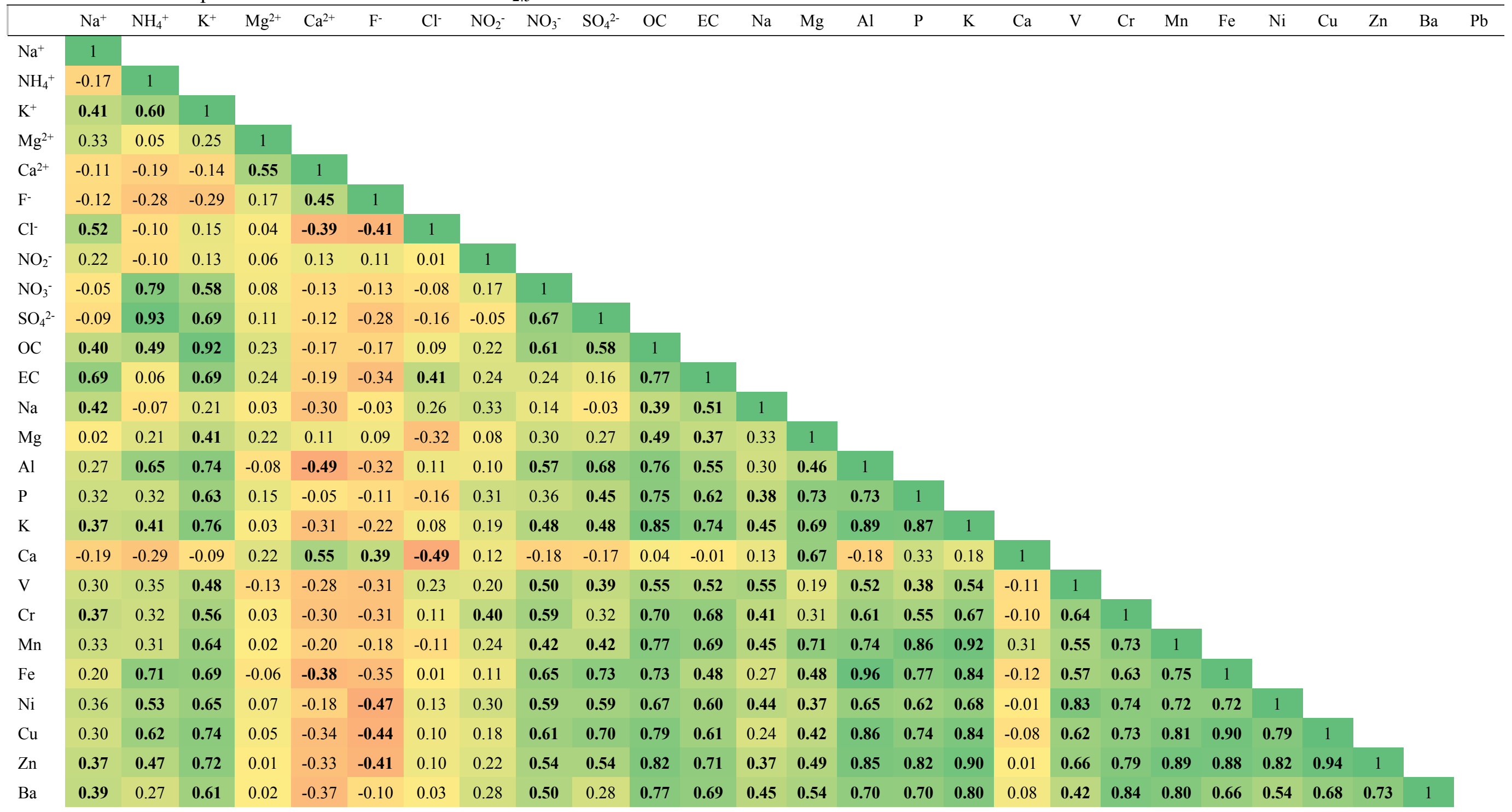




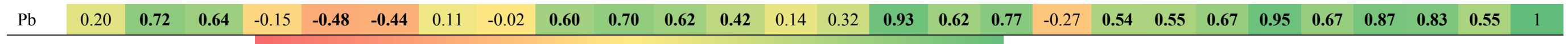
$\begin{array}{llllllllllllllll}\text { Color Key: }-1.00 & 1.00\end{array}$

Bold indicates $p<0.05$. 
Table S4. Percentage changes (mean and 95\% confidence intervals) in CRP associated with interquartile range increases in 24-h average concentrations of $\mathrm{PM}_{2.5}$ constituents.

\begin{tabular}{cccc}
\hline Metallic elements & Model 1 & Model 2 & Model 3 \\
\hline $\mathrm{Na}$ & $-6.9(-18.6,6.5)$ & $-4.5(-16.6,9.5)$ & $-1.2(-13.6,13.0)$ \\
$\mathrm{Mg}$ & $-5.4(-17.5,8.5)$ & $14.8(-6.6,41.2)$ & $7.0(-12.4,30.8)$ \\
$\mathrm{Al}$ & $0.1(-25.6,34.6)$ & $\mathbf{4 9 . 9}(\mathbf{1 . 0}, \mathbf{1 2 2 . 6})$ & $\mathbf{5 3 . 2}(\mathbf{1 5 . 8}, \mathbf{1 0 2 . 6 )}$ \\
$\mathrm{P}$ & $-9.6(-23.5,6.9)$ & $\mathbf{6 7 . 6}(\mathbf{7 . 8}, \mathbf{1 6 0 . 4 )}$ & $25.4(-16.1,87.3)$ \\
$\mathrm{K}$ & $-14.3(-28.6,3.0)$ & $3.7(-24.1,41.7)$ & $14.2(-15.2,54.0)$ \\
$\mathrm{Ca}$ & $9.9(-12.6,38.1)$ & $26.3(-1.3,61.7)$ & $11.9(-11.4,41.3)$ \\
$\mathrm{V}$ & $-3.3(-10.0,4.0)$ & $-1.6(-8.6,5.9)$ & $-0.2(-7.2,7.4)$ \\
$\mathrm{Cr}$ & $\mathbf{- 1 1 . 3 ( - 1 9 . 3 , - 2 . 5 )}$ & $-8.4(-18.8,3.3)$ & $-9.5(-19.8,2.0)$ \\
$\mathrm{Mn}$ & $-5.4(-14.4,4.6)$ & $22.0(-1.3,50.7)$ & $10.8(-9.4,35.5)$ \\
$\mathrm{Fe}$ & $-3.8(-18.4,13.3)$ & $\mathbf{3 6 . 7}(\mathbf{3 . 8}, \mathbf{7 9 . 9 )}$ & $\mathbf{4 4 . 8}(\mathbf{1 1 . 1}, \mathbf{8 8 . 8})$ \\
$\mathrm{Ni}$ & $-7.1(-15.7,2.5)$ & $-3.0(-13.0,8.2)$ & $-1.6(-11.8,9.7)$ \\
$\mathrm{Cu}$ & $-8.7(-22.3,7.3)$ & $\mathbf{6 9 . 6}(\mathbf{1 1 . 6 , 1 5 7 . 9 )}$ & $\mathbf{8 0 . 2}(\mathbf{2 7 . 0}, \mathbf{1 5 5 . 7})$ \\
$\mathrm{Zn}$ & $-12.9(-25.3,1.7)$ & $18.4(-21.9,79.4)$ & $6.2(-29.3,59.5)$ \\
$\mathrm{Ba}$ & $\mathbf{- 1 5 . 8 ( - 2 8 . 3 , - 1 . 2 )}$ & $-6.3(-32.4,29.9)$ & $-11.4(-35.9,22.5)$ \\
$\mathrm{Pb}$ & $6.9(-13.1,31.5)$ & $\mathbf{2 7 . 1}(\mathbf{0 . 4}, \mathbf{6 0 . 9})$ & $\mathbf{3 4 . 6}(\mathbf{9 . 4}, \mathbf{6 5 . 7})$ \\
\hline $\mathrm{Ab}$
\end{tabular}

Abbreviations: CRP, C-reactive protein; Model 1, single-constituent model; Model 2, constituent-PM ${ }_{2.5}$ joint model; Model 3, constituent-residual model. All three models adjusted for gender, age, body mass index, exercise times, relative humidity, temperature, and time trend. Bold indicates $p<0.05$. 
Table S5. Percentage changes (mean and 95\% confidence intervals) in MCP-1 associated with interquartile range increases in 24-h average concentrations of $\mathrm{PM}_{2.5}$ constituents.

\begin{tabular}{|c|c|c|c|}
\hline Metallic elements & Model 1 & Model 2 & Model 3 \\
\hline $\mathrm{Na}$ & $-0.4(-3.2,2.4)$ & $-0.3(-3.1,2.6)$ & $-0.1(-2.9,2.7)$ \\
\hline $\mathrm{Mg}$ & $-2.0(-4.8,0.8)$ & $-3.3(-7.4,1.0)$ & $-3.4(-7.3,0.7)$ \\
\hline $\mathrm{Al}$ & $1.5(-4.5,8.0)$ & $5.0(-3.4,14.2)$ & $3.7(-2.3,10.0)$ \\
\hline $\mathrm{P}$ & $0.6(-2.8,4.2)$ & $13.1(2.7,24.6)$ & $8.8(-0.2,18.6)$ \\
\hline K & $-4.0(-7.6,-0.2)$ & $-9.1(-14.9,-3.0)$ & $-7.9(-13.5,-1.9)$ \\
\hline $\mathrm{Ca}$ & $-5.8(-10.1,-1.2)$ & $-6.2(-10.9,-1.3)$ & $-5.9(-10.3,-1.3)$ \\
\hline V & $1.9(0.4,3.4)$ & $2.1(0.6,3.7)$ & $2.1(0.6,3.7)$ \\
\hline $\mathrm{Cr}$ & $-4.4(-6.3,-2.6)$ & $-6.6(-8.9,-4.3)$ & $-6.6(-8.8,-4.3)$ \\
\hline $\mathrm{Mn}$ & $-1.0(-3.0,1.1)$ & $-2.2(-6.6,2.4)$ & $-2.3(-6.4,2.0)$ \\
\hline $\mathrm{Fe}$ & $2.0(-1.4,5.6)$ & $8.7(2.5,15.2)$ & $8.5(2.6,14.7)$ \\
\hline $\mathrm{Ni}$ & $1.8(-0.3,3.8)$ & $2.5(0.2,4.9)$ & $2.6(0.3,4.9)$ \\
\hline $\mathrm{Cu}$ & $-0.6(-3.9,2.8)$ & $1.9(-7.1,11.8)$ & $2.4(-5.1,10.6)$ \\
\hline $\mathrm{Zn}$ & $-2.2(-5.3,1.0)$ & $-10.7(-18.6,-2.0)$ & $-10.6(-18.3,-2.2)$ \\
\hline $\mathrm{Ba}$ & $-4.6(-7.7,-1.4)$ & $-15.5(-20.9,-9.6)$ & $-15.3(-20.7,-9.5)$ \\
\hline $\mathrm{Pb}$ & $3.8(-0.6,8.4)$ & $6.0(0.8,11.3)$ & $5.2(0.7,9.9)$ \\
\hline
\end{tabular}

Abbreviations: MCP-1, monocyte chemoattractant protein-1; Model 1, single-constituent model; Model 2, constituent- $\mathrm{PM}_{2.5}$ joint model; Model 3, constituent-residual model. All three models adjusted for gender, age, body mass index, exercise times, relative humidity, temperature, and time trend. Bold indicates $p<$ 0.05 . 
Table S6. Percentage changes (mean and 95\% confidence intervals) in sVCAM-1 associated with interquartile range increases in 24-h average concentrations of $\mathrm{PM}_{2.5}$ constituents.

\begin{tabular}{|c|c|c|c|}
\hline Metallic elements & Model 1 & Model 2 & Model 3 \\
\hline $\mathrm{Na}$ & $5.1(2.2,8.1)$ & $7.2(4.4,10.1)$ & $9.0(6.1,12.0)$ \\
\hline $\mathrm{Mg}$ & $-12.3(-14.5,-10.0)$ & $-12.7(-16.1,-9.2)$ & $-15.2(-18.4,-11.7)$ \\
\hline $\mathrm{Al}$ & $-16.2(-21.2,-11.0)$ & $-6.6(-13.8,1.3)$ & $11.2(4.7,18.0)$ \\
\hline $\mathrm{P}$ & $-10.4(-13.4,-7.3)$ & $0.0(-9.1,9.9)$ & $-12.6(-19.9,-4.6)$ \\
\hline K & $-14.6(-17.6,-11.5)$ & $-14.7(-19.9,-9.2)$ & $-7.9(-13.7,-1.8)$ \\
\hline $\mathrm{Ca}$ & $-16.2(-19.9,-12.3)$ & $-12.6(-16.7,-8.3)$ & $-16.1(-19.9,-12.1)$ \\
\hline V & $0.8(-0.7,2.4)$ & $2.0(0.5,3.5)$ & $2.8(1.3,4.4)$ \\
\hline $\mathrm{Cr}$ & $-7.6(-9.3,-5.8)$ & $-5.9(-8.1,-3.6)$ & $-6.6(-8.9,-4.3)$ \\
\hline $\mathrm{Mn}$ & $-8.0(-9.8,-6.2)$ & $-9.8(-13.7,-5.8)$ & $-13.2(-16.8,-9.5)$ \\
\hline $\mathrm{Fe}$ & $-9.6(-12.6,-6.5)$ & $-2.0(-7.4,3.8)$ & $3.1(-2.7,9.3)$ \\
\hline $\mathrm{Ni}$ & $-1.6(-3.6,0.4)$ & $1.8(-0.4,4.1)$ & $2.6(0.3,5.0)$ \\
\hline $\mathrm{Cu}$ & $-10.5(-13.4,-7.5)$ & $-4.1(-12.4,4.9)$ & $12.4(4.0,21.4)$ \\
\hline $\mathrm{Zn}$ & $-12.5(-15.1,-9.8)$ & $-24.2(-30.7,-17.2)$ & $-29.1(-35.1,-22.5)$ \\
\hline $\mathrm{Ba}$ & $-13.2(-15.9,-10.5)$ & $-17.0(-22.2,-11.5)$ & $-19.7(-24.9,-14.2)$ \\
\hline $\mathrm{Pb}$ & $-10.7(-14.4,-6.8)$ & $-5.2(-9.6,-0.5)$ & $2.9(-1.7,7.6)$ \\
\hline
\end{tabular}

Abbreviations: Model 1, single-constituent model; Model 2, constituent-PM ${ }_{2.5}$ joint model; Model 3, constituent-residual model; sVCAM-1, soluble vascular cell adhesion molecule-1. All three models adjusted for gender, age, body mass index, exercise times, relative humidity, temperature, and time trend. Bold indicates $p<0.05$. 
Table S7. Percentage changes (mean and 95\% confidence intervals) in sICAM-1 associated with interquartile range increases in 24-h average concentrations of $\mathrm{PM}_{2.5}$ constituents.

\begin{tabular}{cccc}
\hline Metallic elements & Model 1 & Model 2 & Model 3 \\
\hline $\mathrm{Na}$ & $0.5(-1.8,2.9)$ & $0.3(-2.1,2.7)$ & $0.0(-2.3,2.4)$ \\
$\mathrm{Mg}$ & $0.3(-2.1,2.7)$ & $-1.5(-5.1,2.1)$ & $-0.9(-4.3,2.6)$ \\
$\mathrm{Al}$ & $1.2(-4.0,6.5)$ & $-1.2(-8.0,6.0)$ & $-2.4(-7.2,2.6)$ \\
$\mathrm{P}$ & $1.9(-1.0,5.0)$ & $4.7(-4.5,14.7)$ & $5.6(-2.4,14.3)$ \\
$\mathrm{K}$ & $0.6(-2.6,3.9)$ & $-2.4(-7.8,3.4)$ & $-3.0(-8.2,2.5)$ \\
$\mathrm{Ca}$ & $-1.6(-5.5,2.4)$ & $-2.9(-7.0,1.4)$ & $-1.8(-5.7,2.3)$ \\
$\mathrm{V}$ & $0.9(-0.3,2.2)$ & $0.8(-0.5,2.1)$ & $0.7(-0.6,2.0)$ \\
$\mathrm{Cr}$ & $0.0(-1.7,1.6)$ & $-0.9(-3.0,1.2)$ & $-0.8(-2.9,1.3)$ \\
$\mathrm{Mn}$ & $0.7(-1.1,2.4)$ & $-0.9(-5.0,3.3)$ & $0.0(-3.8,3.9)$ \\
$\mathrm{Fe}$ & $1.6(-1.3,4.5)$ & $1.1(-3.9,6.4)$ & $0.3(-4.5,5.4)$ \\
$\mathrm{Ni}$ & $1.4(-0.3,3.1)$ & $1.2(-0.7,3.2)$ & $1.1(-0.8,3.0)$ \\
$\mathrm{Cu}$ & $1.4(-1.4,4.3)$ & $0.1(-8.3,9.3)$ & $-2.1(-8.7,5.1)$ \\
$\mathrm{Zn}$ & $1.3(-1.4,4.1)$ & $-0.6(-9.4,9.0)$ & $0.9(-7.6,10.2)$ \\
$\mathrm{Ba}$ & $0.5(-2.3,3.3)$ & $-3.5(-9.1,2.4)$ & $-2.9(-8.4,3.0)$ \\
$\mathrm{Pb}$ & $1.4(-2.2,5.2)$ & $0.6(-3.6,5.0)$ & $-0.4(-4.1,3.4)$ \\
\hline $\mathrm{Abbr}$ &
\end{tabular}

Abbreviations: Model 1, single-constituent model; Model 2, constituent-PM ${ }_{2.5}$ joint model; Model 3, constituent-residual model; sICAM-1, soluble intercellular adhesion molecule-1. All three models adjusted for gender, age, body mass index, exercise times, relative humidity, temperature, and time trend. 
Table S8. Percentage changes (mean and 95\% confidence intervals) in ET-1 associated with interquartile range increases in 24-h average concentrations of $\mathrm{PM}_{2.5}$ constituents.

\begin{tabular}{|c|c|c|c|}
\hline Metallic elements & Model 1 & Model 2 & Model 3 \\
\hline $\mathrm{Na}$ & $-4.4(-8.9,0.3)$ & $-3.3(-7.8,1.5)$ & $-1.7(-6.2,3.2)$ \\
\hline $\mathrm{Mg}$ & $-5.4(-9.9,-0.6)$ & $-0.1(-7.2,7.5)$ & $-2.9(-9.6,4.3)$ \\
\hline $\mathrm{Al}$ & $-4.0(-13.6,6.6)$ & $11.7(-2.6,28.1)$ & $17.4(6.5,29.4)$ \\
\hline $\mathrm{P}$ & $-8.5(-13.7,-2.9)$ & $-4.8(-16.9,9.0)$ & $-10.5(-21.2,1.7)$ \\
\hline K & $-8.8(-14.5,-2.7)$ & $-4.2(-13.7,6.4)$ & $0.6(-9.0,11.3)$ \\
\hline $\mathrm{Ca}$ & $-9.2(-16.3,-1.5)$ & $-5.5(-13.5,3.2)$ & $-8.9(-16.2,-1.0)$ \\
\hline V & $-0.7(-3.2,1.9)$ & $0.1(-2.4,2.8)$ & $0.8(-1.8,3.5)$ \\
\hline $\mathrm{Cr}$ & $-7.7(-10.7,-4.6)$ & $-7.5(-11.3,-3.6)$ & $-8.0(-11.8,-4.0)$ \\
\hline $\mathrm{Mn}$ & $-5.0(-8.3,-1.6)$ & $-2.5(-8.8,4.3)$ & $-4.9(-10.9,1.5)$ \\
\hline $\mathrm{Fe}$ & $-4.1(-9.5,1.6)$ & $7.4(-2.1,17.9)$ & $10.9(1.4,21.3)$ \\
\hline $\mathrm{Ni}$ & $-2.8(-6.2,0.6)$ & $-0.7(-4.4,3.3)$ & $0.0(-3.9,4.0)$ \\
\hline $\mathrm{Cu}$ & $-8.8(-13.8,-3.5)$ & $-7.7(-18.8,4.8)$ & $2.6(-8.2,14.7)$ \\
\hline $\mathrm{Zn}$ & $-9.2(-14.0,-4.2)$ & $-11.5(-21.6,-0.1)$ & $-13.5(-23.4,-2.5)$ \\
\hline $\mathrm{Ba}$ & $-10.1(-15.0,-4.9)$ & $-12.0(-21.2,-1.7)$ & $-13.7(-22.8,-3.7)$ \\
\hline $\mathrm{Pb}$ & $-0.3(-7.3,7.2)$ & $6.6(-1.8,15.6)$ & $10.6(3.0,18.9)$ \\
\hline
\end{tabular}

Abbreviations: ET-1, endothelin-1; Model 1, single-constituent model; Model 2, constituent-PM ${ }_{2.5}$ joint model; Model 3, constituent-residual model. All three models adjusted for gender, age, body mass index, exercise times, relative humidity, temperature, and time trend. Bold indicates $p<0.05$. 


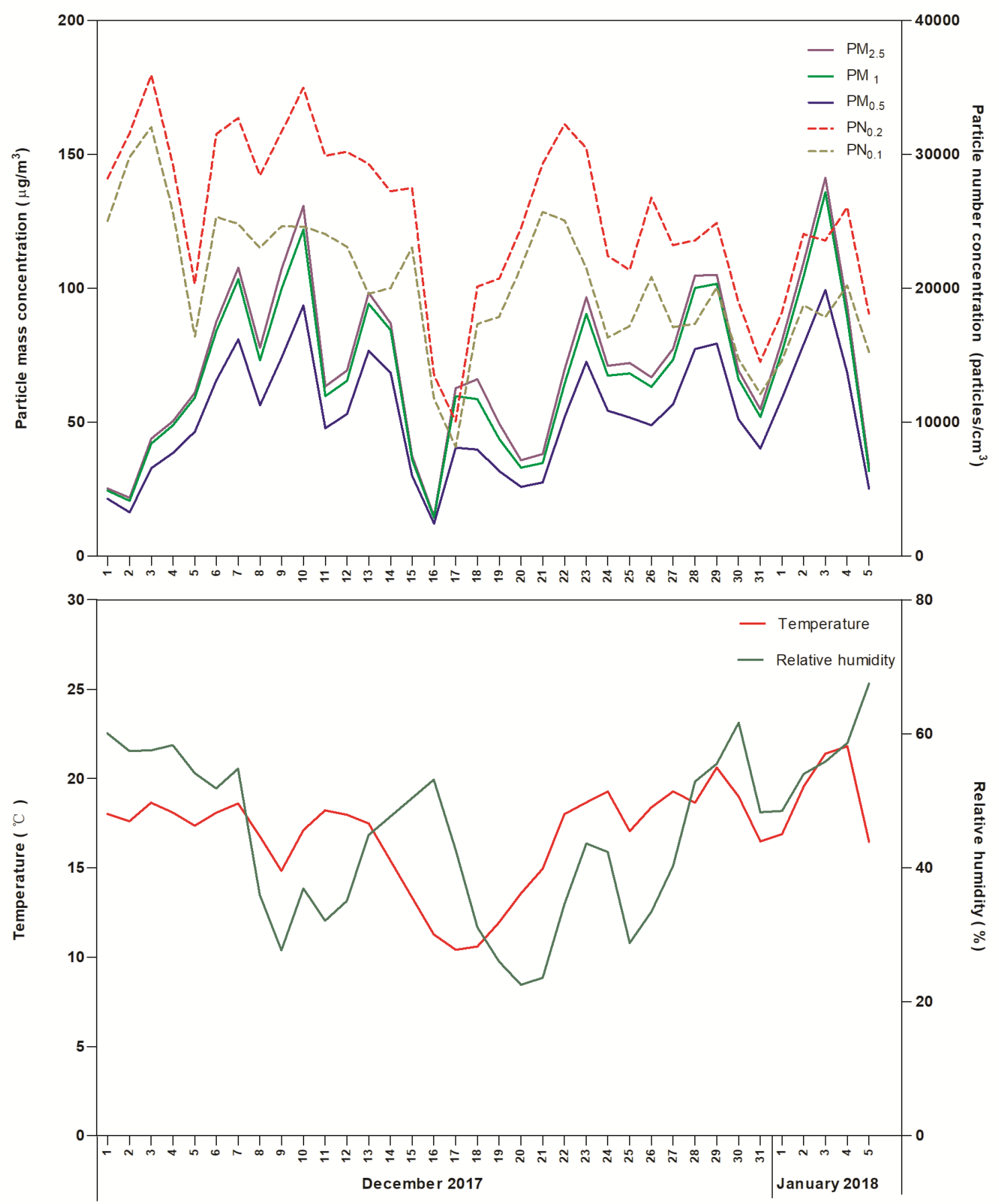

Figure S1. Time series of size-fractionated PM and weather conditions in Guangzhou, China, between December 1, 2017 and January 5, 2018. 